\title{
Robust Coordinated Tracking Control of Multiple Robots System Under Bounded Inputs
}

\author{
Song GAO ${ }^{1,2}$, Rui SONG ${ }^{1,2, *}$, Yukun ZHENG ${ }^{1,2}$, Yibin LI $^{1,2}$ \\ ${ }^{1}$ Center for Robotics, School of Control Science and Engineering, Shandong University, \\ No. 17923, Jingshi Road, Jinan, 250061, Shandong, China \\ ${ }^{2}$ Engineering Research Center of Intelligent Unmanned System, Ministry of Education, \\ No. 17923, Jingshi Road, Jinan, 250061, Shandong, China \\ gaosong8286@mail.sdu.edu.cn, rsong@sdu.edu.cn (*Corresponding author), \\ zhengyk@mail.sdu.edu.cn, liyb@sdu.edu.cn
}

\begin{abstract}
The coordinated tracking control of multiple robots system with input saturation and obstacle avoidance is investigated in this paper. Additionally, a robust hierarchical outer-inner layer controller is proposed. In order to avoid obstacles and realize coordination tracking control, null-space-based behavioural (NSB) control is applied in the outer layer where it generates the velocity required by the inner layer. An improved adaptive radical basis function neural networks (RBFNNS) proportional derivative-sliding mode control (IRPD-SMC) method is proposed in the inner layer to achieve robust tracking, null steady-state errors, and bounded inputs. Finally, in an environment with obstacles, all robots can track the target at a fixed distance and distribute around it evenly. The convergence and stability of the system are certified by Lyapunov stability theory. Simulation results in the 2D and 3D space show that the proposed IRPD-SMC is effective, by comparing the performances of this method with those of proportional derivative-sliding mode control (PD-SMC), adaptive proportional derivative-sliding mode control (APD-SMC), and adaptive sliding mode control (ASMC).
\end{abstract}

Keywords: Multiple robots system, NSB, RBFNNs, IRPD-SMC, Bounded inputs.

\section{Introduction}

Recently, coordination tracking control problem has been favoured by many researchers due to its application value, especially in formation mission and entrapment or escorting mission (Ma et al., 2019). Owing to measurement noise, modelling errors, external disturbances, and modelling simplifications, unmodelled dynamics often exist in practical systems, which can severely degrade the performance of the system and cause unstable control systems. In addition, disregarding the practical issues such as input saturation and obstacles may have serious consequences. Therefore, it is necessary to study these issues with regard to coordination tracking control.

Obviously, the requirement to maintain accurate relative distances or orientations between robots is essential and imperative in the coordination tracking control. Until now, many methods have been used to achieve coordination tracking control, e.g., cyclic pursuit strategy (Yu \& Liu, 2016), behavior-based methods (Zhou \& Xia, 2015), and cluster space control method (Mas et al., 2009).

When compared with other behavioral methods, the NSB control exhibits an obvious feature, i.e., a clear mathematical representation. In (Schlanbusch et al., 2011), a behavioral control solution using the NSB control and sliding mode control was presented to achieve reconfiguration and avoid collisions in spacecraft formations.
In (Zhou \& Xia, 2015), a cooperation control strategy was designed to complete the escorting mission in the environments with obstacles, and the system is proved to be convergent in finitetime. However, it requires a certain knowledge of system parameters discussed in the above mentioned specialized literatures, while in many practices it is difficult to obtain accurately (Kara \& Mary, 2017, Yue et al., 2016). In (Zhang et al., 2018), the APD-SMC method was employed to enhance the capability of 2D overhead crane systems as it is simple and robust. A robust model-free inner-outer layer controller scheme was proposed in (Gao et al., 2019), which had the advantages of simplicity and robustness.

Recently, many researchers have begun to use many learning-based methods to identify models needed to enhance the performance of tradition control methods. Neural networks are adopted to model nonlinear unknown functions owing to their excellent approximation abilities. In (Li et al., 2016), RBFNNs was used to estimate the function which is unknown, and finally the uniform and bounded tracking error was realized. In (Zhou et al., 2019), the universal approximation feature of RBFNNs which was adopted in controller design, overcame the design difficulties caused by nonlinearity and uncertainty.

Input saturation may deteriorate the performance of multiple robots system up to an unacceptable 
level; therefore, scholars have studied many antisaturation control strategies (Guo et al., 2019, Sun et al., 2020, Chen et al., 2018). In (Lu et al., 2019), an arctangent function was designed in a control signal to ensure that the input was bounded to prevent actuator saturation. In (Guo et al., 2019), two robust antisaturation controllers were designed based on the saturation function to address the input constraint.

Motivated by (Lu et al., 2019) and (Li et al., 2019), a robust hierarchical NSB+IRPD-SMC controller is proposed herein. In comparison with the current existing control algorithms, this paper has made the following contributions. Specifically speaking, the first aspect is that the proposed NSB+IRPD-SMC controller exhibits characteristics of simplicity, strong robustness, and is model-free. Secondly, this controller has a strong learning ability, does not require complex calculation, and achieves null steady-state errors. Finally, it can guarantee the boundedness of the control torques and prevent the actuator saturation without affecting the stability of the system.

This paper is organized as follows. Section 2 presents the system models and several properties. Section 3 describes the outer layer controller design in detail. Section 4 presents the inner layer controller and analyses the stability of the system. The results of the experiments under different conditions and some resolutions are detailed in Section 5 and 6, respectively.

\section{System Model}

A system consisting of $n$ robots expressed by Euler-Lagrange equation is described below.
$M_{i}\left(q_{i}\right) \ddot{q}_{i}+C_{i}\left(q_{i}, \dot{q}_{i}\right) \dot{q}_{i}+g_{i}\left(q_{i}\right)+\tau_{i}^{d}=\tau_{i}$,

$i=1, \ldots, n$

where $M_{i}\left(q_{i}\right) \in \mathbb{R}^{p \times p}, q_{i} \in \mathbb{R}^{p}, C_{i}\left(q_{i}, \dot{q}_{i}\right) \dot{q}_{i} \in \mathbb{R}^{p}$, $g_{i}\left(q_{i}\right), \tau_{i}$ and $\tau_{i}^{d}$ represent the inertia matrix, the generalized coordinates vector, the centrifugalCoriolis vector, the gravity term, the control torque input of the $i$ th robot, and the unknown disturbance vector, respectively.

The following properties used in this paper are given:

Property 2.1. Skew symmetry.

$x^{T}\left[\dot{M}_{i}\left(q_{i}\right) / 2-C_{i}\left(q_{i}, \dot{q}_{i}\right)\right] x=0, \forall x \in \mathbb{R}^{p}$.

Property 2.2. The system model is linearly parameterized.

$M_{i}\left(q_{i}\right) x+C_{i}\left(q_{i}, \dot{q}_{i}\right) y+g_{i}\left(q_{i}\right)=Y_{i}\left(q_{i}, \dot{q}_{i}, x, y\right) \Theta_{i}$

$Y_{i}\left(q_{i}, \dot{q}_{i}, x, y\right)$ is the dynamic regression matrix,

$\Theta_{i}$ is a vector associated with the $i$ th robot which contains the unknown constant parameters, and $x, y \in \mathbb{R}^{p}$.

Property 2.3. Boundedness.

$M_{i}\left(q_{i}\right), C_{i}\left(q_{i}, \dot{q}_{i}\right), g_{i}\left(q_{i}\right)$ and $\tau_{i}^{d}$ are all bounded.

\section{Outer Layer Controller Design}

NSB control is applied in the outer layer to incorporate the three different tasks in order to form the coordination tracking motion with obstacle avoidance and generate the required velocity for the robots. IRPD-SMC is proposed in inner layer to remove the influences aroused by model uncertainties and disturbances and ensure the boundedness of the inputs. The entire control system diagram is illustrated in Figure 1.

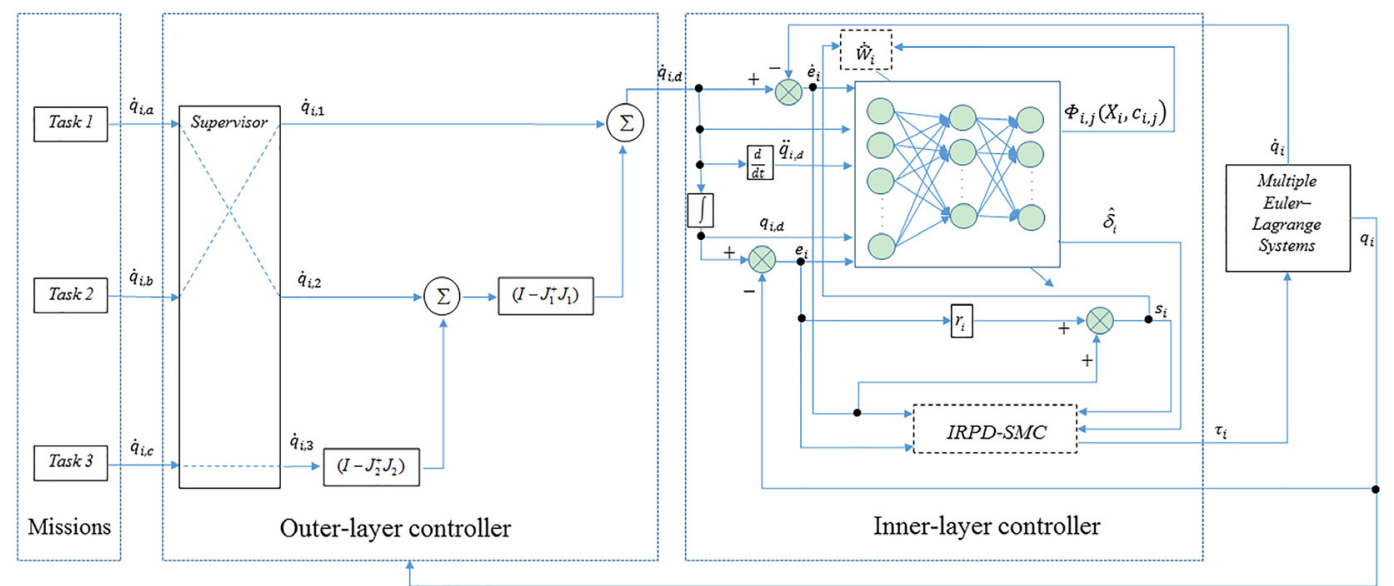

Figure 1. Control structure 
Three different tasks are considered in this section for the coordination tracking and obstacle avoidance, and the desired velocity is

$\dot{q}_{d}=\dot{q}_{1}+\left(I-J_{1}^{\dagger} J_{1}\right)\left[\dot{q}_{2}+\left(I-J_{2}^{+} J_{2}\right) \dot{q}_{3}\right]$

$\dot{q}_{d}=\left[\dot{q}_{1, d}^{T}, \ldots \dot{q}_{n, d}^{T}\right]^{T} \in \mathbb{R}^{p n}, \dot{q}_{1}=\left[\dot{q}_{1,1}^{T}, \ldots \dot{q}_{n, 1}^{T}\right]^{T} \in \mathbb{R}^{p n}$

represents the ideal velocity of avoiding obstacles (task 1), $\dot{q}_{2}=\left[\dot{q}_{1,2}^{T}, \ldots \dot{q}_{n, 2}^{T}\right]^{T} \in \mathbb{R}^{p n}$ is the desired velocity of distributing evenly around a target (task 2), $\dot{q}_{3}=\left[\dot{q}_{1,3}^{T}, \ldots \dot{q}_{n, 3}^{T}\right]^{T} \in \mathbb{R}^{p n}$ is the desired velocity of maintaining on a sphere/ hypersphere surface (task 3).

$\dot{q}_{i, 1}=J_{i, 1}^{\dagger} \chi_{i, 1} \tilde{f}_{i, 1}=\chi_{i, 1}\left(\max \left\{d_{i}-\left\|q_{i}-q_{i}^{o}\right\|, 0\right\}\right) \hat{r}_{i}$,

where the error function of task 1 is

$\tilde{f}_{i, 1}=f_{i, 1 d}-f_{i, 1}=d_{i}-\max \left\{\left\|q_{i}-q_{i}^{o}\right\|, d_{i}\right\}$

$q_{i}^{o}$ indicates the obstacle position of robot $i$; $d_{i}$ is the specified allowable safety distance from the robot $i$ to the obstacle; the Jacobian matrix is $J_{i, 1}=\left(q_{i}-q_{i}^{o}\right)^{T} /\left\|q_{i}-q_{i}^{o}\right\| ; \quad \chi_{i, 1}>0$ is a gain which will be given later, and $J_{i, 1}^{\dagger}=J_{i, 1}^{T}$, $J_{1}=\left[J_{1,1}, \ldots J_{n, 1}\right] \in \mathbb{R}^{1 \times p n}$.

Using the planar case as example in task 2, the desired formation is an $n$ regular polygon and all robots remain on the vertices eventually.

$\dot{q}_{2}=J_{2}^{\dagger} \Lambda_{2} \tilde{f}_{2}$, where $\Lambda_{2} \in \mathbb{R}^{n \times n}$ represents a gains matrix which is constant positive-definite,

$J_{2}=$ block $\operatorname{diag}\left\{\left(q_{k_{1}}-q_{k_{n}}\right)^{T}, \ldots\left(q_{k_{j}}-q_{k_{j-1}}\right)^{T}, \ldots\right\}$

$\in \mathbb{R}^{n \times p n}$

is the Jacobian matrix, whose pseudoinverse is

$J_{2}^{\dagger}=$ block $\operatorname{diag}\left\{\left(q_{k_{1}}-q_{k_{n}}\right) /\left(q_{k_{1}}-q_{k_{n}}\right)^{T}\left(q_{k_{1}}-q_{k_{n}}\right), \ldots\right.$

$\left.\left(q_{k_{j}}-q_{k_{j-1}}\right) /\left(q_{k_{j}}-q_{k_{j-1}}\right)^{T}\left(q_{k_{j}}-q_{k_{j-1}}\right), \ldots\right\} \in \mathbb{R}^{p n \times n}$

The error function of task 2 is defined as

$\tilde{f}_{2}=f_{2 d}-f_{2}$

$=\left[l_{i}^{2} / 2, \ldots l_{i}^{2} / 2\right]^{T}-\left[\frac{1}{2}\left(q_{k_{1}}-q_{k_{n}}\right)^{T}\left(q_{k_{1}}-q_{k_{n}}\right), \ldots\right.$

$\left.\frac{1}{2}\left(q_{k_{j}}-q_{k_{j-1}}\right)^{T}\left(q_{k_{j}}-q_{k_{j-1}}\right), \ldots\right]^{T}$

where $q_{k_{j}}=\left[q_{k_{j}, 1} \ldots q_{k_{i}, p}\right]^{T} \in \mathbb{R}^{p} ; k_{j}$ is the $j$ th position of the robot on the circle, but it is not the $j$ th robots.
In task $3, \dot{q}_{3}=J_{3}^{\dagger} \Lambda_{3} \tilde{f}_{3}$ and the error function is

$\tilde{f}_{3}=f_{3 d}-f_{3}$

$=\left[R^{2} / 2, \ldots R^{2} / 2\right]^{T}-\left[\left(q_{1}-q_{t}\right)^{T}\left(q_{1}-q_{t}\right) / 2, \ldots\right.$

$\left.\left(q_{n}-q_{t}\right)^{T}\left(q_{n}-q_{t}\right) / 2\right]^{T}$

where $q_{t}$ is the position of the target;

$J_{3}=$ block $\operatorname{diag}\left\{\left(q_{1}-q_{t}\right)^{T}, \ldots\left(q_{n}-q_{t}\right)^{T}\right\} \in \mathbb{R}^{n \times p n} ;$

$\Lambda_{3} \in \mathbb{R}^{n \times n}$, with a similar definition as the one of $\Lambda_{2}$, is also a gain matrix which is constant positive- definite;

$J_{3}^{\dagger}=$ block $\operatorname{diag}\left\{\left(q_{1}-q_{t}\right) /\left(q_{1}-q_{t}\right)^{T}\left(q_{1}-q_{t}\right), \ldots\right.$

$\left.\left(q_{n}-q_{t}\right) /\left(q_{n}-q_{t}\right)^{T}\left(q_{n}-q_{t}\right)\right\} \in \mathbb{R}^{p n \times n}$

\section{Inner Layer Controller Design}

To address the model uncertainties and external disturbances as well as to achieve null steady-state errors tracking and bounded inputs, IRPD-SMC is proposed for the inner layer.

\subsection{IRPD-SMC Law}

The expected trajectory tracked by each robot is $q_{d}(t)=\left[q_{1, d}^{T}(t), \ldots q_{n, d}^{T}(t)\right]^{T} \in \mathbb{R}^{p n}$, it is obtained via integration of $\dot{q}_{d}(t)$ in equation (2), and the definition of $\ddot{q}_{d}(t)$ is similar. As both of them are bounded. Define the following sliding surface

$s_{i}=\dot{q}_{i, r}-\dot{q}_{i}=\dot{q}_{i, d}-\dot{q}_{i}+r_{i} e_{i}=\dot{e}_{i}+r_{i} e_{i}$

where

$s_{i}=\left[s_{i, 1}, \ldots s_{\mathrm{i}, p}\right]^{T} \in \mathbb{R}^{p}, \dot{q}_{i, r}=\left[\dot{q}_{\mathrm{i}, r 1}, \ldots \dot{q}_{i, r p}\right]^{T} \in \mathbb{R}^{p}$ isthe nominal reference states and $r_{i}=\operatorname{diag}\left(r_{i, 1}, r_{i, 2} \ldots r_{i, p}\right)$ is the sliding constant, which is positive. Position and velocity tracking errors are

$e_{i}=q_{i, d}(t)-q_{i}(t)=\left[e_{i, 1}, \ldots e_{i, p}\right]^{T} \in \mathbb{R}^{p}$ and

$\dot{e}_{i}=\dot{q}_{i, d}(t)-\dot{q}_{i}(t)=\left[\dot{e}_{i, 1}, \ldots \dot{e}_{i, p}\right]^{T} \in \mathbb{R}^{p}$, respectively.

According to Property 2.2, the nominal reference torque is obtained below

$M_{i}\left(\mathrm{q}_{i}\right) \ddot{\mathrm{q}}_{i, r}+C_{i}\left(q_{i}, \dot{q}_{i}\right) \dot{q}_{i, r}+g_{i}\left(q_{i}\right)+\tau_{i}^{d}$

$=Y_{i}\left(q_{i}, \dot{q}_{i}, \dot{q}_{i, r}, \ddot{q}_{i, r}\right) \Theta_{i}=\delta_{i}$

As has been shown, $\delta_{i}=\left[\delta_{i, 1}, \ldots, \delta_{i, p}\right]^{T} \in \mathbb{R}^{p}$ is unknown and it is hard to determine its value 
because it has disturbances and uncertain dynamics. Therefore, it is proposed to utilize the approximation properties of RBFNNS to estimate the unknown term $\delta_{i}$.

As illustrated in Figure 1, the adaptive RBFNNS is written as

$\hat{\delta}_{i}=\hat{W}_{i}^{T} \Phi_{i}\left(X_{i}\right)$

where the input is $X_{i}=\left[\mathrm{x}_{i, 1}, \mathbf{x}_{i, 2} \ldots \mathbf{x}_{i, m}\right]^{T}, W_{i} \in \mathbb{R}^{v^{*} p}$ is the weight of the network,

$v$ is the nodes number of neurons,

$\Phi_{i}\left(X_{i}\right)=\left[\Phi_{i, 1}\left(X_{i}, \mathrm{c}_{i, 1}\right), \ldots \Phi_{i, v}\left(X_{i}, \mathrm{c}_{i, v}\right)\right]^{T} \in \mathbb{R}^{v}$ and

$\Phi_{i, j}\left(X_{i}, \mathrm{c}_{i, j}\right)(j=1, \ldots v)$ is the activation function. Gaussian function is a typically activation function, expressed as

$\Phi_{i, j}\left(X_{i}, \mathrm{c}_{i, j}\right)=\exp \left(-\left\|X_{i}-c_{i, j}\right\|^{2} / \sigma_{i, j}^{2}\right)$

where $\mathrm{c}_{i, j}$ and $\sigma_{i, j}$ are the center and breadth of the $j$ th network.

The optimal RBFNNS for learning $\delta_{i}$ is

$\delta_{i}=\left(W_{i}^{*}\right)^{T} \Phi_{i}\left(X_{i}\right)+\varepsilon_{i}$

where $W_{i}^{*} \in \mathbb{R}^{\nu^{*} p}$ is the optimal value of the weight vector; $\varepsilon_{i}$ is the bounded approximation error.

The adaptive RBFNNS is written as

$\hat{\delta}_{i}=\hat{W}_{i}^{T} \Phi_{i}\left(X_{i}\right)$

the updating law of weight is

$\dot{\hat{W}}_{i}=\mu_{i} \Phi_{i}\left(X_{i}\right) s_{i}^{T}$

where the gain $\mu_{i}$ is a diagonal matrix which is positive-definite.

Using equations (8) and (10), one obtains

$\tilde{\delta}_{i}=\delta_{i}-\hat{\delta}_{i}=\tilde{W}_{i}^{T} \Phi_{i}\left(X_{i}\right)+\varepsilon_{i}$

$\tilde{W}_{i}=W_{i}^{*}-\hat{W}_{i}$. In this study, the input of the RBFNNS is selected as $X_{i}=\left[e_{i}, \dot{e}_{i}, q_{i, d}, \dot{q}_{i, d}, \ddot{q}_{i, d}\right]^{T}$. Using the estimation term $\hat{\delta}_{i}$, RPD-SMC law is expressed as follows:

$\tau_{i}=\hat{\delta}_{i}+k_{p i} e_{i}+k_{d i} \dot{e}_{i}+k_{i} \operatorname{sign}\left(s_{i}\right)$

Obviously, the PD part is utilized to substitute the equivalent control of SMC; the robust part is utilized to ensure robustness, and the RBFNNS part is used to approximate the uncertain term. Finally, the system can achieve null steady-state errors tracking. However, in order solve the inputs constraint, the RPD-SMC law must be improved.

Compared with hyperbolic tangent, the arctangent function $\arctan \left(\kappa_{i} x\right)$ has a wider range of $\left(-\frac{\pi}{2}, \frac{\pi}{2}\right)$ than that of the function $\tanh \left(\kappa_{i} x\right)$ with a range of $(-1,1)$. With the same value of $\kappa_{i}$, which is a constant, $\arctan \left(\kappa_{i} x\right)$ approaches saturation more moderately. The smaller the value of $\kappa_{i}$, the smaller the zero-crossing slope of function $\arctan \left(\kappa_{i} x\right)$ is, and this function can approach saturation almost linearly. To describe the proportional relationship more effectively, $\arctan \left(\kappa_{i} x\right)$ is often multiplied by a certain amount of gain, i.e., $\lambda_{i} \arctan \left(\kappa_{i} x\right)$. Therefore, the IRPD-SMC strategy is

$\tau_{i}=\hat{\delta}_{i}+k_{\alpha i} \lambda_{i} \arctan \left(\kappa_{i} e_{i}\right)+k_{\beta i} \lambda_{i} \arctan \left(\kappa_{i} \dot{e}_{i}\right)$
$+k_{i} \operatorname{sign}\left(s_{i}\right)$

where $k_{\alpha i}$ is the position error-related gain that eliminates the positioning error; $k_{\beta i}$ is the velocity error-related gain which eliminates the velocity tracking error; $\lambda_{i}$ is the approximate proportional gain; $k_{i}$ is the robust term gain. All gains are positive definite.

\subsection{Stability Analysis}

Applying equations (6) and (7) into equation (1), the equation is obtained as follows:

$M_{i}\left(q_{i}\right) \dot{s}_{i}+C_{i}\left(q_{i}, \dot{q}_{i}\right) s_{i}+\tau_{i}=\delta_{i}, i=1, \ldots, n$

Theorem 4.1. Considering multiple robots system described in equation (1), and the IRPD-SMC law and the weight update law as shown in equations (15) and (12). Assuming that Properties 2.1-2.3 hold, provided the control gains satisfy $k_{i}>\varepsilon_{i, \max }$, and regardless of disturbances and the uncertainties, the following results have been obtained.

1. Outer-inner layer system is asymptotically steady with errors $e_{i}, \dot{e}_{i}$ converging to 0 , if no conflict exists among the three tasks.

2. If task 1 is active and conflicts with task 2 and 3, $\chi_{i, 1}\left(\left\|e_{i}\right\|,\left\|\dot{q}_{i}\right\|\right)=\chi_{i, 1}^{*}\left(\left\|e_{i}\right\|,\left\|\dot{q}_{i}\right\|\right)+\varrho_{i}$, and $\varrho_{i}$ is a robust term devised to reject noise, then task 1 is executed first. Finally, outerinner layer system is asymptotically steady with errors $e_{i}, \dot{e}_{i}$ converging to 0 . 
Proof. Select the following Lyapunov functions

$$
\begin{aligned}
& V=V_{1}+V_{2} \\
& V_{1}=\frac{1}{2} \sum_{i=1}^{n} s_{i}^{T} \kappa_{i} M_{i} s_{i}+\sum_{i=1}^{n} k_{\alpha i} \lambda_{i}\left\{\kappa_{i} e_{i} \arctan \left(\kappa_{i} e_{i}\right)\right. \\
& \left.-\frac{1}{2} \ln \left[1+\left(\kappa_{i} e_{i}\right)^{2}\right]\right\}+\frac{1}{2} \sum_{i=1}^{n} \operatorname{tr}\left(\tilde{W}_{i}^{T} \mu_{i}^{-1} \tilde{W}_{i}\right) \\
& V_{2}=\frac{1}{2}\left(\tilde{f}_{1}^{T} \eta_{1} \tilde{f}_{1}+\tilde{f}_{2}^{T} \eta_{2} \tilde{f}_{2}+\tilde{f}_{3}^{T} \eta_{3} \tilde{f}_{3}\right)
\end{aligned}
$$

where $\eta_{1}, \eta_{2}$, and $\eta_{3}$ are design parameters which are positive-definite and $\tilde{f}_{1}=\sum_{i=1}^{n} \tilde{f}_{i, 1}$.

By substituting the IRPD-SMC law (15) into equation (16), it results

$M_{i}\left(q_{i}\right) \dot{s}_{i}=\tilde{\delta}_{i}-C_{i}\left(q_{i} \dot{q}_{i}\right) s_{i}-k_{\alpha i} \lambda_{i} \operatorname{art} \tan \left(\kappa_{i} e_{i}\right)$

$-k_{\beta i} \lambda_{i} \operatorname{art} \tan \left(\kappa_{i} \dot{e}_{i}\right)-k_{i} \operatorname{sign}\left(s_{i}\right)$

Differentiating $V_{1}$ and substituting equations (6), (12), and (18) into it, one obtains

$\dot{V}_{1}=\sum_{i=1}^{n} s_{i}^{T} \kappa_{i} M_{i}\left(q_{i}\right) \dot{s}_{i}+\sum_{i=1}^{n} \frac{1}{2} s_{i}^{T} \kappa_{i} \dot{M}_{i}\left(q_{i}\right) s_{i}$

$+\sum_{i=1}^{n} k_{\alpha i} \lambda_{i} \kappa_{i} \dot{e}_{i} \arctan \left(\kappa_{i} e_{i}\right)-\sum_{i=1}^{n} \kappa_{i} \mu_{i}^{-1} \operatorname{tr}\left(\tilde{W}_{i}^{T} \dot{\hat{W}}_{i}\right)$

$\leq \sum_{i=1}^{n} \kappa_{i}\left\|s_{i}^{T}\right\|\left(\varepsilon_{i, \max }-k_{i}\right)-\sum_{i=1}^{n} \dot{e}_{i}^{T} \kappa_{i} k_{\beta i} \lambda_{i} \arctan \left(\kappa \dot{e}_{i}\right)$

$-\sum_{i=1}^{n} r_{i} \kappa_{i} e_{i}^{T} k_{\alpha i} \lambda_{i} \arctan \left(\kappa e_{i}\right)-\sum_{i=1}^{n} \gamma_{i} \kappa_{i} e_{i}^{T} k_{\beta i} \lambda_{i} \arctan \left(\kappa \dot{e}_{i}\right)$

If $k_{i}>\varepsilon_{i, \max }$, that is, $\sum^{n}\left\|s_{i}^{T}\right\|\left(\varepsilon_{i, \max }-k_{i}\right)$ is negative definite, then $\dot{V}_{1}<0$.Thus, the errors $e_{i}, \dot{e}_{i}$ will converge asymptotically to 0 and the inner layer system is globally stable.

Remark 4.1. In order to eliminate chattering which is caused by $\operatorname{sign}(\cdot)$ function, a hyperbolic tangent function is introduced and equation (15) is modified into

$\tau_{i}=\hat{\delta}_{i}+k_{\alpha i} \lambda \arctan \left(e_{i}\right)+k_{\beta i} \lambda \arctan \left(\dot{e}_{i}\right)+k_{i} \tanh \left(s_{i}\right)$

where $\tanh \left(s_{i}\right)=e_{i}^{s_{i}}-e_{i}^{-s_{i}} / e_{i}^{s_{i}}+e_{i}^{-s_{i}}$.

Calculating the derivative of $V_{2}$ and substituting equations (3-5) into it, it yields
$\dot{V}_{2}=-\eta_{1} \tilde{f}_{1}^{T} \dot{f}_{1}-\eta_{2} \tilde{f}_{2}^{T} \dot{f}_{2}-\tilde{f}_{3}^{T} \eta_{3} \dot{f}_{3}$

$=-\tilde{f}_{1}^{T} \eta_{1} J_{1} \dot{q}_{d}-\tilde{f}_{2}^{T} \eta_{2} J_{2} \dot{q}_{d}-\tilde{f}_{3}^{T} \eta_{3} J_{3} \dot{q}_{d}$

$=-\tilde{f}_{1}^{T} \eta_{1} \lambda_{1} \tilde{f}_{1}-\tilde{f}_{2}^{T} \eta_{2} \Lambda_{2} \tilde{f}_{2}-\tilde{f}_{3}^{T} \eta_{3} \Lambda_{3} \tilde{f}_{3}$

$-\tilde{f}_{2}^{T} \eta_{2} \lambda_{1} J_{2} J_{1}^{+} \tilde{f}_{1}-\tilde{f}_{3}^{T} \eta_{3} \lambda_{1} J_{3} J_{1}^{+} \tilde{f}_{1}$

$-\tilde{f}_{3}^{T} \eta_{3} \Lambda_{2} J_{3} J_{2}^{+} \tilde{f}_{2}+\tilde{f}_{2}^{T} \eta_{2} \Lambda_{2} J_{2} J_{1}^{+} J_{1} J_{2}^{+} \tilde{f}_{2}$

$+\tilde{f}_{2}^{T} \eta_{2} J_{2} J_{1}^{+} J_{1} J_{3}^{+} \Lambda_{3} \tilde{f}_{3}+\tilde{f}_{3}^{T} \eta_{3} J_{3} J_{2}^{+} J_{2} J_{3}^{+} \Lambda_{3} \tilde{f}_{3}$

$+\tilde{f}_{3}^{T} \eta_{3} \Lambda_{2} J_{3} J_{1}^{+} J_{1} J_{2}^{+} \tilde{f}_{2}+\tilde{f}_{3}^{T} \eta_{3} J_{3} J_{1}^{+} J_{1} J_{3}^{+} \Lambda_{3} \tilde{f}_{3}$

$-\tilde{f}_{2}^{T} \eta_{2} J_{2} J_{1}^{+} J_{1} J_{2}^{+} J_{2} J_{3}^{+} \Lambda_{3} \tilde{f}_{3}$

$-\tilde{f}_{3}^{T} \eta_{3} J_{3} J_{1}^{+} J_{1} J_{2}^{+} J_{2} J_{3}^{+} \Lambda_{3} \tilde{f}_{3}$

If no conflict exists in the three tasks, then $J_{2} J_{1}^{+}=0, J_{3} J_{1}^{+}=0, J_{3} J_{2}^{+}=0$. Therefore, equation (21) yields

$\dot{V}_{2}=-\tilde{f}_{1}^{T} \eta_{1} \lambda_{1} \tilde{f}_{1}-\tilde{f}_{2}^{T} \eta_{2} \Lambda_{2} \tilde{f}_{2}-\tilde{f}_{3}^{T} \eta_{3} \Lambda_{3} \tilde{f}_{3} \leq 0$

It can be noticed that the NSB system is globally stable.

Assuming that task 1 is active and conflicts with task 2 and task $3, \dot{V}_{2}$ could be rewritten as

$\dot{V}_{2}=-X^{T} M X$

where $X=\left[\tilde{f}_{1}^{T}, \tilde{f}_{2}^{T}, \tilde{f}_{3}^{T}\right]^{T}, M=\left[m_{i j}\right], i, j=1,2,3$

with $m_{11}=\eta_{1} \chi_{1}, m_{21}=\eta_{2} \chi_{1} J_{2} J_{1}^{+}$,

$m_{22}=\eta_{2} \Lambda_{2}-\eta_{2} \Lambda_{2} J_{2} J_{1}^{+} J_{1} J_{2}^{+}$,

$m_{23}=-\eta_{2} J_{2} J_{1}^{+} J_{1} J_{3}^{+} \Lambda_{3}+\eta_{2} J_{2} J_{1}^{+} J_{1} J_{2}^{+} J_{2} J_{3}^{+} \Lambda_{3}$,

$m_{31}=\eta_{3} \lambda_{1} J_{3} J_{1}^{+}, m_{32}=\eta_{3} \Lambda_{2} J_{3} J_{2}^{+}-\eta_{3} \Lambda_{2} J_{3} J_{1}^{+} J_{1} J_{2}^{+}$,

$m_{33}=\eta_{3} \Lambda_{3}-\eta_{3} J_{3} J_{2}^{+} J_{2} J_{3}^{+} \Lambda_{3}-\eta_{3} J_{3} J_{1}^{+} J_{1} J_{3}^{+} \Lambda_{3}$

$+\eta_{3} J_{3} J_{1}^{+} J_{1} J_{2}^{+} J_{2} J_{3}^{+} \Lambda_{3}$

Furthermore, $2|a b| \leq a^{2}+b^{2}$ for any $a, b \in \mathbb{R}$ is applied in equation (23) in order to yield

$$
\begin{aligned}
& X^{T} M X \geq\left(m_{11, l}-m_{12, L}-m_{13, L}\right)\left\|\tilde{f}_{1}\right\|^{2} \\
& +\left(m_{22, l}-m_{21, L}-m_{22, L}\right)\left\|\tilde{f}_{2}\right\|^{2} \\
& +\left(m_{33, l}-m_{31, L}-m_{32, L}\right)\left\|\tilde{f}_{3}\right\|^{2}
\end{aligned}
$$

where $m_{i j, l}$ and $m_{i j, L}$ represent the lower and upper limits of $m_{i j}$, respectively.

$$
X^{T} P X \geq \frac{1}{2}\left(m_{11, l}\left\|\tilde{f}_{1}\right\|^{2}+m_{22, l}\left\|\tilde{f}_{2}\right\|^{2}+m_{33, l}\left\|\tilde{f}_{3}\right\|^{2}\right)
$$

because $\left\|J_{1}\right\|=\left\|J_{2}\right\|=\left\|J_{3}\right\|=1, \quad m_{22, l}=m_{22, L}=0$, $m_{33, l}=m_{33, L}=0, f_{2}$ and $f_{3}$ are losses of control, then $\dot{q}_{d}=\dot{q}_{1}$. Therefore, $V_{2}$ can be reselected as $V_{2}=\frac{1}{2} \tilde{f}_{1}^{T} \eta_{1} \tilde{f}_{1}$. Subsequently, $\dot{V}_{2}=-\tilde{f}_{1}^{T} \eta_{1} \chi_{1} \tilde{f}_{1} \leq 0$ is obtained. In this case, the outer layer system is also globally stable. 
Inserting equation (2) into equation (6), it results

$\left(\dot{q}_{i}-J_{i, 1}^{+} \chi_{i, 1} \tilde{f}_{i, 1}\right)^{T} k_{\alpha i}\left(\dot{q}_{i}-J_{i, 1}^{+} \chi_{i, 1} \tilde{f}_{i, 1}\right)$

$>k_{\alpha i} e_{i}^{T} r_{i}^{2} e_{i}+k_{\alpha i} \gamma_{i} e_{i}\left(\dot{q}_{i}-J_{i, 1}^{+} \chi_{i, 1} \tilde{f}_{i, 1}\right)^{T}$

$+k_{\alpha i} r_{i} e_{i}^{T}\left(\dot{q}_{i}-J_{i, 1}^{+} \chi_{i, 1} \tilde{f}_{i, 1}\right)$

Taking the norm on both sides of equation (25) and manipulating it as an equality form, one obtains

$\chi_{i, 1}^{*}\left(\left\|e_{i}\right\|,\left\|\dot{q}_{i}\right\|\right)=b_{i}+\sqrt{b_{i}^{2}+4 a_{i} c_{i}} / 2 a_{i}$

where $a_{i}=\tilde{f}_{i, 1}^{2}, b_{i}=2 \tilde{f}_{i, 1}\left(\left\|\dot{q}_{i}\right\|-2 r_{i}\left\|e_{i}\right\|\right)$,

$c_{i}=\gamma_{i}^{2}\left\|e_{i}\right\|^{2}+2 r_{i}\left\|e_{i}\right\|\left\|\dot{q}_{i}\right\|-\left\|\dot{q}_{i}\right\|^{2}$.

By selecting $\chi_{i, 1}\left(\left\|e_{i}\right\|,\left\|\dot{q}_{i}\right\|\right)=\chi_{i, 1}^{*}\left(\left\|e_{i}\right\|,\left\|\dot{q}_{i}\right\|\right)+\varrho_{i}$,

where $\varrho_{i}>0$, robots can successfully avoid obstacles.

To summarize, whenever any conflict exists in the three tasks or not, the whole system must be globally stable, $e_{i}$ and $\dot{e}_{i}$ eventually become 0 .

Remark 4.2. If there is only a conflict between task 2 and task 3 , each robot will only perform task 1 and task 2, namely, $\dot{q}_{d}=\dot{q}_{1}+\left(I-J_{1}^{+} J_{1}\right) \dot{q}_{2}$, and no conflict will happen.

\section{Simulation experiments}

Several simulation experiments are conducted to display the superior capability of IRPD-SMC compared with that of APD-SMC (Gao et al., 2019), ASMC (Zhou \& Xia, 2015), and PDSMC(Ouyang et al., 2014).

Consider five robots and six robots in the 2D space and 3D space, respectively; assume that $M_{i}=1$, $C_{i}=0$ and $g_{i}=0$. The parameters of the outer layer in the 2D and 3D space are shown in Tables 1 and 2, respectively. The control parameters of ASMC, PD-SMC, APD-SMC, and of the proposed IRPD-SMC are displayed in Table 3, and they have been adjusted through trial and error.

Table 1. Parameters of outer layer in the 2D space.

\begin{tabular}{|l|l|}
\hline Symbol & Value \\
\hline$R$ & 5 \\
\hline$d_{i}$ & 2 \\
\hline$l_{i}$ & $2 R \cos (\pi / 2-\pi / n)$ \\
\hline$\Lambda_{2}, \Lambda_{3}$ & $\operatorname{diag}(2 \ldots 2)$ \\
\hline$\varrho_{i}$ & 0.1 \\
\hline
\end{tabular}

Table 2. Parameters of outer layer in the 3D space

\begin{tabular}{|l|l|}
\hline Symbol & Value \\
\hline$R$ & 5 \\
\hline$d_{i}$ & 2 \\
\hline$l_{i}$ & $\sqrt{2} R$ \\
\hline$\Lambda_{2}, \Lambda_{3}$ & $\operatorname{diag}(2 \ldots 2)$ \\
\hline$\varrho_{i}$ & 0.1 \\
\hline
\end{tabular}

Table 3. Control parameters of different controllers

\begin{tabular}{|c|c|}
\hline Controller & Value \\
\hline ASMC & $k_{i}=5$ \\
\hline \multirow{3}{*}{ PD-SMC } & $k_{p i}=\operatorname{diag}(5 \ldots 5)$ \\
\hline & $k_{d i}=\operatorname{diag}(6 \ldots 6)$ \\
\hline & $k_{i}=\operatorname{diag}(10 \ldots 10)$ \\
\hline \multirow{6}{*}{ APD-SMC } & $k_{p i}=\operatorname{diag}(5 \ldots 5)$ \\
\hline & $k_{d i}=\operatorname{diag}(6 \ldots 6)$ \\
\hline & $k_{i}=\operatorname{diag}(1 \ldots 1)$ \\
\hline & $r=\operatorname{diag}(4 \ldots 4)$ \\
\hline & $\mu_{i}=\operatorname{diag}(2 \ldots 2)$ \\
\hline & $\hat{\rho}_{i, 0}=[0.1 \ldots 0.1]^{T}$ \\
\hline \multirow{9}{*}{ IRPD-SMC } & $k_{\alpha i}=\operatorname{diag}(5 \ldots . .5)$ \\
\hline & $k_{\beta i}=\operatorname{diag}(6 \ldots 6)$ \\
\hline & $\lambda_{i}=\operatorname{diag}(5.5 \ldots 5.5)$ \\
\hline & $k_{i}=\operatorname{diag}(1 \ldots 1)$ \\
\hline & $\kappa_{i}=0.2$ \\
\hline & $r=\operatorname{diag}(4 \ldots 4)$ \\
\hline & $\mu_{i}=\operatorname{diag}(2 \ldots 2)$ \\
\hline & $\sigma_{i, j}=25$ \\
\hline & $\begin{array}{l}c_{i, j}=[-3,-2,-1,0,1,2,3] \\
j=1,2, \ldots, 7\end{array}$ \\
\hline
\end{tabular}

Case 1. In the $2 \mathrm{D}$ space, initial values for the positions of five robots are $q_{1}(0)=[5,10]^{T}$, $q_{2}(0)=[-5,5]^{T}, \quad q_{3}(0)=[-5,-5]^{T}, q_{4}(0)=[5,-10]^{T}$, and $q_{5}(0)=[5,0]^{T}$. The trajectory of the target is defined as $c=[3+0.1 t, 0]^{T}$. The external disturbance parameters are:

$\tau_{i}^{d}=\left\{\begin{array}{c}\tau_{i}^{d 1}(t \leq 40) \\ \tau_{i}^{d 2}(\mathrm{else})\end{array}(N), \tau_{i}^{d 1}=1 / 2[\sin (0.5 t), \sin (0.7 t)]^{T}\right.$,

and $\tau_{i}^{d 2}=2, i=1, \ldots, 5$. 
The values of the outer and inner layer parameters used for the simulations are shown in Tables 1 and 3, respectively.

The target and the trajectories of the robots in the 2D space, when using IRPD-SMC, are shown in Figure 2.

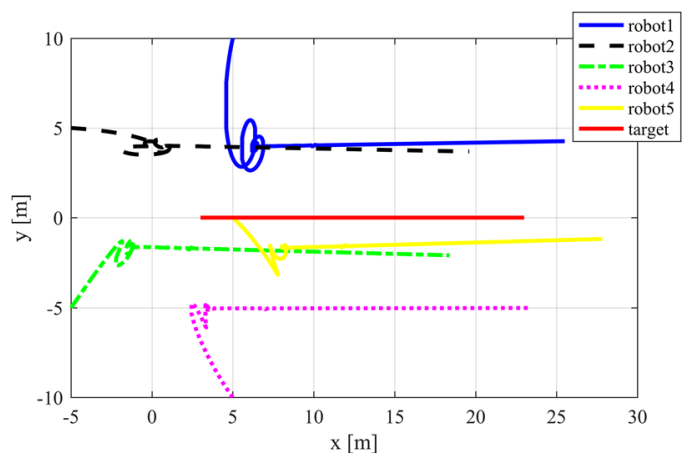

Figure 2. Trajectories of the robots and target using IRPD-SMC

Figures 3-6 illustrate the distances between adjacent robots, the distances between the target and the robot, the position tracking errors, and the velocity tracking errors when using different controllers, respectively. From these figures, it becomes clear that the performances are different, especially when disturbances occur.
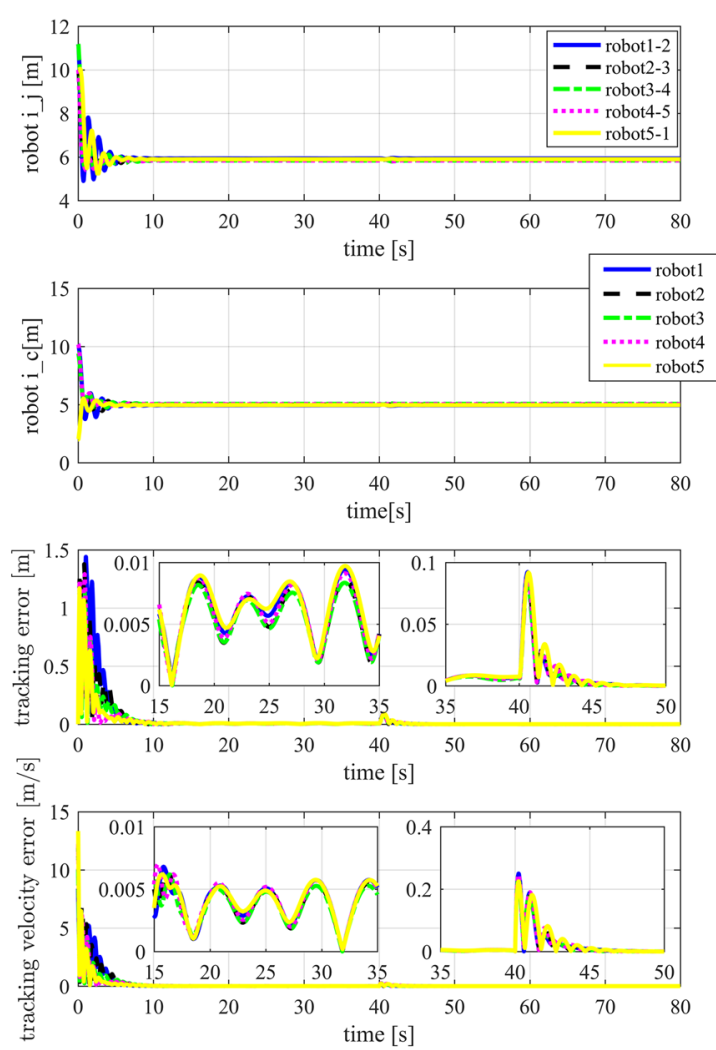

Figure 3. Control results of using IRPD-SMC
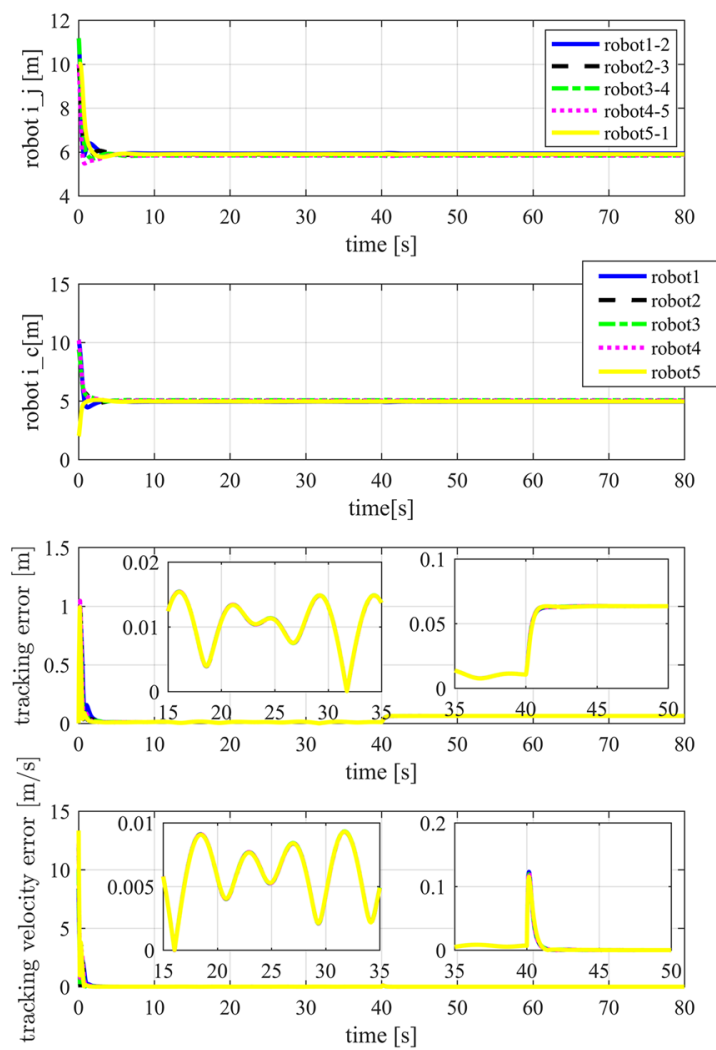

Figure 4. Control results of using PD-SMC
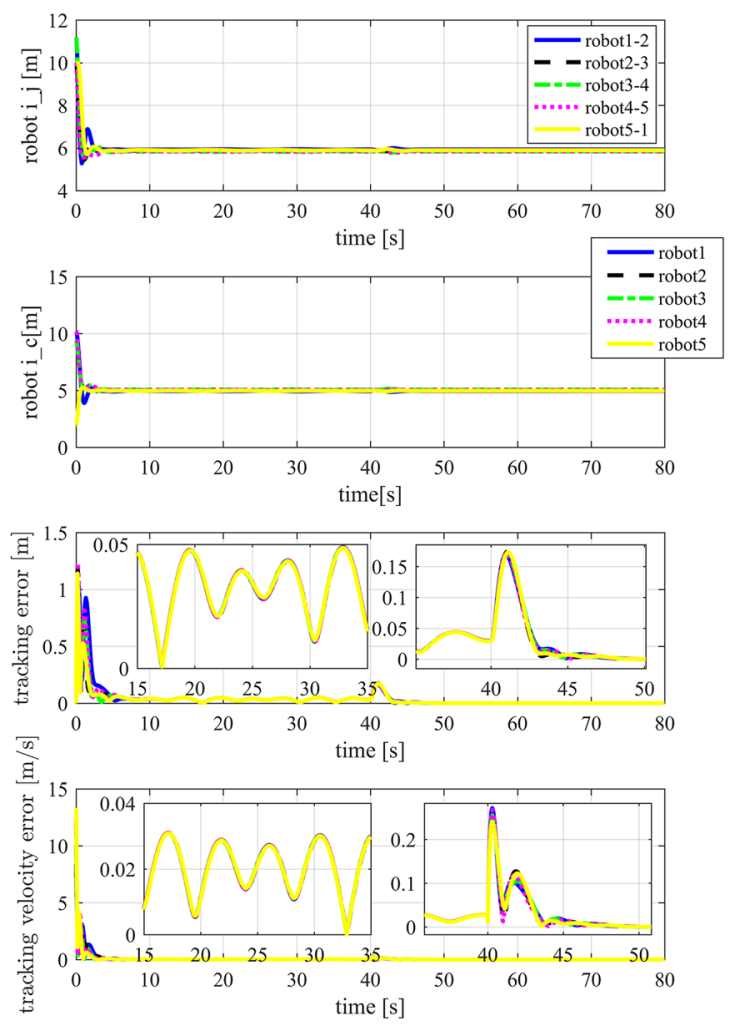

Figure 5. Control results of using APD-SMC 

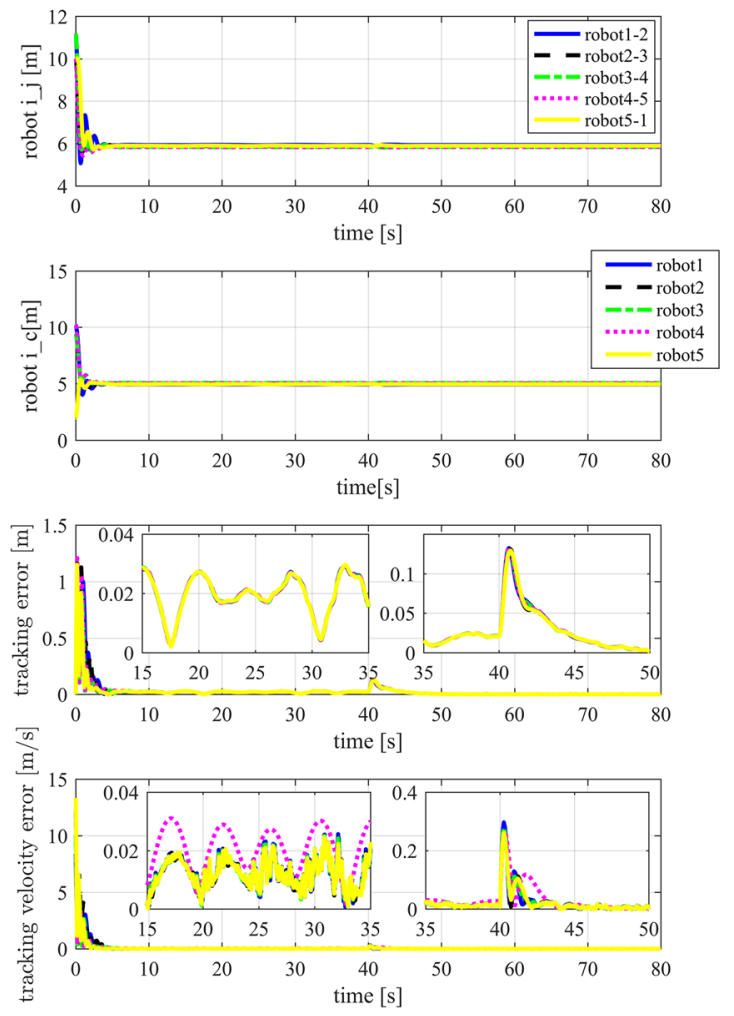

Figure 6. Control results of using ASMC

Figure 3 shows the best performance obtained by using the proposed control algorithm when compared with the one of other controllers.

As demonstrated in Figure 3, disturbances are compensated and the null steady-state errors are achieved.

By contrast, the steady-state errors cannot be well compensated by the PD-SMC controller, as shown in Figure 4. When $\tau_{i}^{d 2}=2(\mathrm{~N})$ are introduced at $t=40 \mathrm{~s}$, tracking errors will always occur in the PD-SMC controller and the robots will never reach the reference trajectories again.

Finally, the control inputs of IRPD-SMC and RPD-SMC represented by equation (14) are illustrated in Figures 7 and 8. As shown, the control inputs have small values that do not exceed $3 \mathrm{~N}$ at the end. However, the initial torque inputs of the RPD-SMC controller are significantly higher than those of IRPD-SMC. This means that controllers without saturation functions usually produce high initial torques, which may even exceed the upper limit of the actuator torques. In addition, it can be noticed that the control torques are continuous and easily physically realizable, which is just the result of using $\tanh (\cdot)$ function.

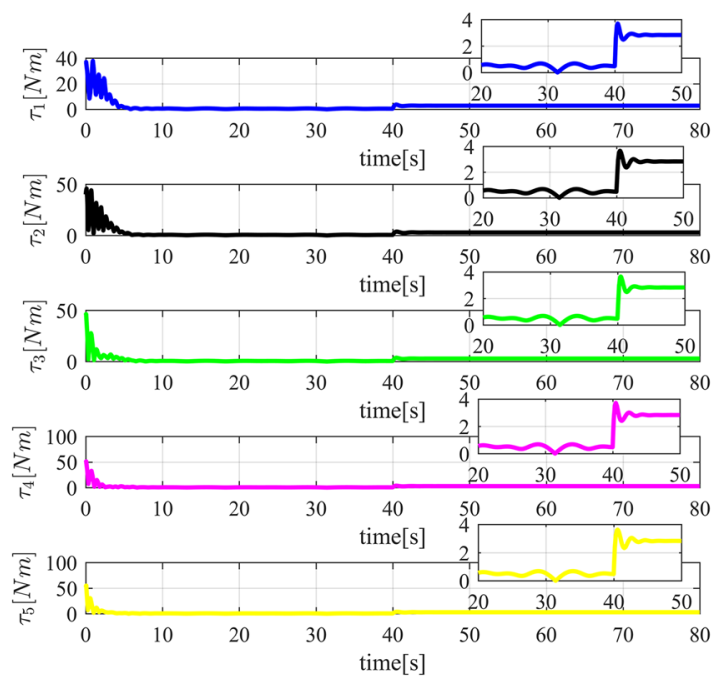

Figure 7. Control torques of using IRPD-SMC
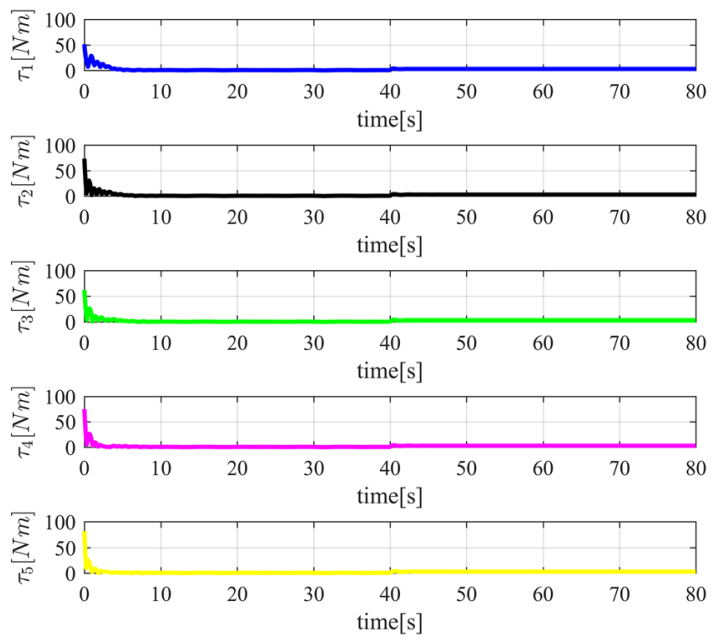

Figure 8. Control torques of using RPD-SMC

Case 2. In the 3D space with Gaussian noise and disturbance, the effectiveness of IRPD-SMC law is examined when the obstacle occurs. The initial positions of six robots are set as $q_{1}(0)=[-10,1,0]^{T}$, $q_{2}(0)=[-1,-10,0.3]^{T}, q_{3}(0)=[10,0,1]^{T}$, $q_{4}(0)=[0,0.5,10]^{T}, \quad q_{5}(0)=[0,10,0.3]^{T}, \quad$ and $q_{6}(0)=[0,0,-10]^{T}$. The trajectory of the target is $c=[0.1 t, 3+0.1 t, \sin 0.1 t]^{T}$ and the position of the obstacle is $q_{0}=[15,3,-4.5]^{T}$. Noise has been considered and simulated with a zero-mean Gaussian function with a standard deviation of 0.2. The disturbance parameters are defined as $\tau_{i}^{d}=1 / 2[\sin (0.5 t), \sin (0.7 t), \cos (0.5 \mathrm{t})]^{T} \quad$ where $i=1, \ldots, 6$. The parameters of the outer layer and the ones of the controller are shown in Tables 2 and 3. 
As shown in Figures 9 and 10, it is clear that the proposed control strategy enables the robots to change their trajectories and avoid obstacles when they appear. In addition, it can effectively reject noise and disturbance.
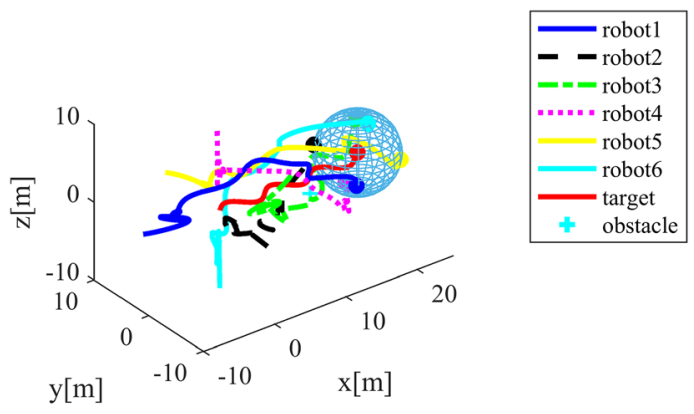

Figure 9. Trajectories of robots and target using IRPD-SMC

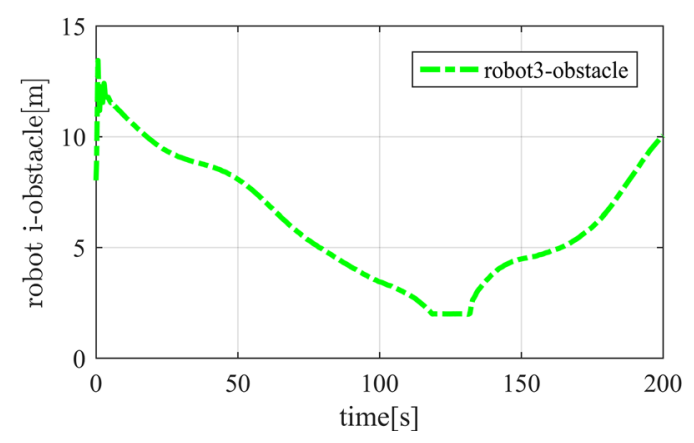

Figure 10. Distance between robot and obstacle using IRPD-SMC

\section{REFERENCES}

Chen, L., Li, C., Sun, Y. \& Ma, G. (2018). Distributed finite-time tracking control for multiple uncertain Euler-Lagrange systems with input saturations and error constraints, IET Control Theory \& Applications, 13(1), 123-133.

Gao, S., Song, R. \& Li, Y. (2019). Coordinated Control of Multiple Euler-Lagrange Systems for Escorting Missions with Obstacle Avoidance, Applied Sciences, 9(19), 4144.

Guo, Y., Huang, B., Li, A. j. \& Wang, C. Q. (2019). Integral sliding mode control for Euler-Lagrange systems with input saturation, International Journal of Robust and Nonlinear Control, 29(9), 1088-1100.

Kara, T. \& Mary, A. H. (2017). Adaptive PD-SMC for Nonlinear Robotic Manipulator Tracking Control. Studies in Informatics and Control, 26(1), 49-58. DOI: 10.24846/v26ily201706

Li, S., Wang, Y., Tan, J. \& Zheng, Y. (2016). Adaptive RBFNNs/integral sliding mode control for a quadrotor aircraft, Neurocomputing, 216, 126-134.

\section{Conclusion}

The coordinated tracking control of multiple robots system has been described in an environment with obstacles, disturbances. A robust hierarchical outer-inner layer control structure composed of NSB and IRPD-SMC has been designed.

Finally, parametric uncertainties and disturbances have been addressed, bounded control inputs have been provided, and fast convergence, robustness, and null steady errors have been obtained.

In addition, the simplicity and model-free features of the proposed controller and continuous control signals have allowed the controller to be easily applied.

The Lyapunov theorems and the results of simulation experiments have verified that the proposed control law is effective. In a future work some discussions about the design of the observer in coordination control will be given.

Li, Z., Ma, X. \& Li, Y. (2019). Robust tracking control strategy for a quadrotor using RPD-SMC and RISE, Neurocomputing, 331, 312-322.

Lu, B., Fang, Y. \& Sun, N. (2019). Nonlinear coordination control of offshore boom cranes with bounded control inputs, International Journal of Robust and Nonlinear Control, 29(9), 1165-1181.

Ma, J., Lu, H., Xiao, J., Zeng, Z. \& Zheng, Z. (2019). Multi-robot Target Encirclement Control with Collision Avoidance via Deep Reinforcement Learning, Journal of Intelligent \& Robotic Systems, 99, 371-386.

Mas, I., Li, S., Acain, J. \& Kitts, C. (2009). Entrapment/ escorting and patrolling missions in multi-robot cluster space control. In 2009 IEEE/RSJ International Conference on Intelligent Robots and Systems (pp. 5855-5861).

Ouyang, P., Acob, J. \& Pano, V. (2014). PD with sliding mode control for trajectory tracking of robotic system, Robotics and Computer-Integrated Manufacturing, 30(2), 189-200. 
Schlanbusch, R., Kristiansen, R. \& Nicklasson, P. J. (2011). Spacecraft formation reconfiguration with collision avoidance, Automatica, 47(7), 1443-1449.

Sun, Y., Dong, D., Qin, H. \& Wang, W. (2020). Distributed tracking control for multiple EulerLagrange systems with communication delays and input saturation, ISA Transactions, 96, 245-254.

Yu, X. \& Liu, L. (2016). Cooperative control for moving-target circular formation of nonholonomic vehicles, IEEE Transactions on Automatic Control, 62(7), 3448-3454.

Yue, W. H., Pano, V., Ouyang, P. R. \& Hu, Y. Q. (2016). Model-independent position domain sliding mode control for contour tracking of robotic manipulator, International Journal of Systems Science, 48(1), 1-10.
Zhang, M., Ma, X., Song, R., Rong, X., Tian, G., Tian, X. \& Li, Y. (2018). Adaptive proportional-derivative sliding mode control law with improved transient performance for underactuated overhead crane systems, IEEE/CAA Journal of Automatica Sinica, 5(3), 683-690.

Zhou, N., Kawano, Y. \& Cao, M. (2019). Neural Network-Based Adaptive Control for Spacecraft Under Actuator Failures and Input Saturations, IEEE transactions on neural networks and learning systems, PP(99), 1-5. DOI: 10.1109/TNNLS.2019.2945920

Zhou, N. \& Xia, Y. (2015). Coordination control of multiple Euler-Lagrange systems for escorting mission, International Journal of Robust and Nonlinear Control, 25(18), 3596-3616. 